\title{
Performance Analysis of a Low-Noise, Highly Linear Distributed Amplifier in 500-nm InP/InGaAs DHBT Technology
}

Shivan, Tanjil; Hossain, Maruf; Doerner, Ralf; Johansen, Tom Keinicke; Yacoub, Hady ; Boppel, Sebastian; Heinrich, Wolfgang; Krozer, Viktor

Published in:

I E E E Transactions on Microwave Theory and Techniques

Link to article, DOI:

10.1109/TMTT.2019.2947664

Publication date:

2019

Document Version

Peer reviewed version

Link back to DTU Orbit

Citation (APA):

Shivan, T., Hossain, M., Doerner, R., Johansen, T. K., Yacoub, H., Boppel, S., Heinrich, W., \& Krozer, V. (2019). Performance Analysis of a Low-Noise, Highly Linear Distributed Amplifier in 500-nm InP/InGaAs DHBT Technology. I E E E Transactions on Microwave Theory and Techniques, 67(12), 5139-5147. https://doi.org/10.1109/TMTT.2019.2947664

\section{General rights}

Copyright and moral rights for the publications made accessible in the public portal are retained by the authors and/or other copyright owners and it is a condition of accessing publications that users recognise and abide by the legal requirements associated with these rights.

- Users may download and print one copy of any publication from the public portal for the purpose of private study or research.

- You may not further distribute the material or use it for any profit-making activity or commercial gain

- You may freely distribute the URL identifying the publication in the public portal 


\title{
Performance Analysis of a Low-Noise, Highly Linear Distributed Amplifier in 500-nm InP/InGaAs DHBT Technology
}

\author{
Tanjil Shivan $^{(}$, Student Member, IEEE, Maruf Hossain ${ }^{(}$, Ralf Doerner ${ }^{(}$, Member, IEEE, \\ Tom K. Johansen, Member, IEEE, Hady Yacoub, Sebastian Boppel, \\ Wolfgang Heinrich $^{\circledR}$, Fellow, IEEE, and Viktor Krozer, Senior Member, IEEE
}

\begin{abstract}
This article is an extension of the previous report on an ultrawideband distributed amplifier (DA) in the InP double heterojunction bipolar transistor (DHBT) technology. With the choice of a tricode unit cell, the Miller capacitance is reduced and a larger bandwidth (BW) is obtained. Measured S-parameters show a 3-dB BW of near de to $>170 \mathrm{GHz}$ with a gain of $12 \mathrm{~dB}$. In addition, the circuit operates in low de bias conditions, thus yielding a low measured noise figure (NF) of $8 \mathrm{~dB}$ over the frequency range of $5-50 \mathrm{GHz}$ and $12 \mathrm{~dB}$ at $135 \mathrm{GHz}$. Within the $5-50-\mathrm{GHz}$ frequency range, the $\mathrm{NF}$ is only $0.5 \mathrm{~dB}$ higher than the NF of a similar DA with cascode unit cell. When the circuit is measured for large-signal condition, it reaches a 1-dB output compression point, $P_{1 \mathrm{~dB}}$, of $8.4 \mathrm{dBm}$ at $150 \mathrm{GHz}$, a saturated output power of approximately $10 \mathrm{dBm}$, and an associated maximum power-added efficiency (PAE) of $6 \%$. This is the best linearity as well as the highest saturated output power and PAE reported at this frequency for DAs. When measured at $20 \mathrm{GHz}$, the circuit shows a $P_{1 \mathrm{~dB}}$, of $9 \mathrm{dBm}$ and output referred third-order intercept point of $22 \mathrm{dBm}$, a saturated output power of $11 \mathrm{dBm}$, and an associated maximum PAE of $6 \%$, thereby proving its uniform large-signal character within the full band. In short, this circuit brings in the unique combination of low NF, highly linear characteristics, and high PAE simultaneously together over large BW. Furthermore, the circuit consumes $180 \mathrm{~mW}$ of dc power only.
\end{abstract}

Index Terms-Distributed amplifier (DA), InP double heterojunction bipolar transistor (DHBT), monolithic microwave integrated circuit (MMIC), traveling-wave amplifier (TWA), ultrawideband low-noise amplifier.

Manuscript received May 3, 2019; revised August 7, 2019 and October 2, 2019; accepted October 6, 2019. This work was supported in part by the Helmholtz Research School on Security Technologies (HRSST), in part by the Leibniz Association through the SAW Project InP THz HBT, and in part by the German BMBF through the Forschungsfabrik Mikroelektronik Deutschland (FMD) Framework under Grant 16FMD02. This article is an expanded version from the IEEE MTT-S International Microwave Symposium (IMS 2019), Boston, MA, USA, June 2-7, 2019. (Corresponding author: Tanjil Shivan.)

T. Shivan, M. Hossain, R. Doerner, H. Yacoub, S. Boppel, and W. Heinrich are with the Ferdinand-Braun-Institut (FBH), 12489 Berlin, Germany (e-mail: tanjil.shivan@fbh-berlin.de).

T. K. Johansen is with the Department of Electrical Engineering, Technical University of Denmark (DTU), 2800 Kongens Lyngby, Denmark.

V. Krozer is with the Physics Department, Ferdinand-Braun-Institut (FBH), Berlin, Germany, and also with Johann Wolfgang Goethe University, 60323 Frankfurt, Germany.

Color versions of one or more of the figures in this article are available online at http://ieeexplore.iee.org.

Digital Object Identifier 10.1109/TMTT.2019.2947664

\section{INTRODUCTION}

$\mathbf{R}$ ECENT trends in high data rate in optical communications, broadband spectroscopic, and broadband measurement systems are pushing the limits of the current state of the art in broadband amplifiers. Highly uniform ultrawideband operation, low deviation from linear phase, high output dynamic range, and high efficiency are the key requirement in these applications. For receiver applications within the aforementioned systems, low-noise characteristic is additionally required over the large bandwidth (BW). Usual low-noise topologies require low bias condition, which is contradictory to achieving high output dynamic range. Therefore, an ultrawideband, highly linear system with low-noise characteristics is a challenge to achieve both from circuit topology and choice of transistor technology.

A useful first approach is to choose a circuit topology that offers gain within higher than an octave BW. As such, distributed amplification has been a promising architecture in monolithic microwave integrated circuits (MMICs), which was first proposed in GaAs MESFET technology [1]. Several reports have demonstrated the implementation of this architecture in various technologies: InP HEMT [2], InP double heterojunction bipolar transistor (DHBT) [3], CMOS [4], and SiGe HBT [5]-[7]. With increased scaling possibilities, amplifiers up to 235-GHz BWs have been presented [2], [3], [8], [9], but with low output power levels. In order to achieve the state-of-the-art small- and large-signal properties, a 500-nm InP DHBT with $f_{\max }$ of $490 \mathrm{GHz}$ can give high-dynamicrange possibilities. Using these devices as subcells in a distributed architecture, ultrawideband, large dynamic range, and low-noise amplifiers can be designed [10].

This article reports an ultrawideband distributed amplifier (DA) that addresses this demand. This article offers a large $\mathrm{BW}$ of $>170 \mathrm{GHz}$, low deviation from linear phase of only $\pm 10^{\circ}$ up to $165 \mathrm{GHz}$, and a noise figure (NF) of $8 \mathrm{~dB}$ when measured between 5 and $50 \mathrm{GHz}$. In terms of large-signal characteristics, the circuit has a measured output power with $1-\mathrm{dB}$ compression point $P_{1 \mathrm{~dB}}=8.4 \mathrm{dBm}$ at $150 \mathrm{GHz}$, a saturated output power $P_{\text {sat }}=10 \mathrm{dBm}$, and an associated maximum power-added efficiency (PAE) of 6\% at $150 \mathrm{GHz}$. These are the highest $P_{1 \mathrm{~dB}}, P_{\text {sat }}$, and PAE values 


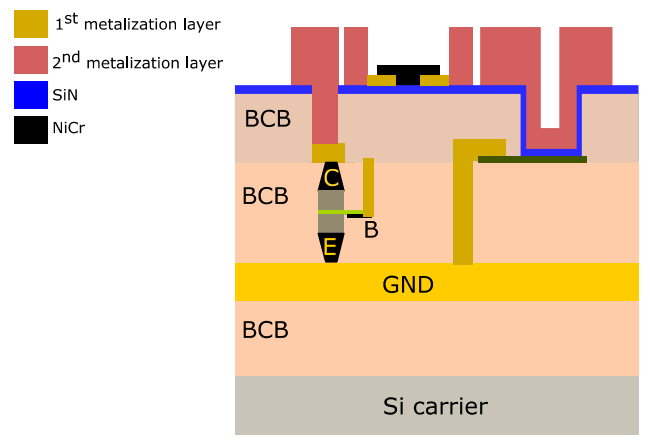

Fig. 1. Cross-sectional schematic of MMIC stack with 500-nm InP DHBT.

reported at these frequencies for amplifiers with BWs larger than $110 \mathrm{GHz}$. The high value of $P_{1 \mathrm{~dB}}$ and PAE is measured at the high end of the BW that indicates similar behavior for the whole frequency range. In terms of noise characteristics, it exhibits among the lowest NF among DAs with BW greater than $110 \mathrm{GHz}$.

This article expands on the authors' previous work [11], with new RF power measurements between 5 and $110 \mathrm{GHz}$, NF measurements from 5 to 50 and from 110 to $150 \mathrm{GHz}$, transistor modeling, and circuit design steps in detail. This article is arranged as follows. Section II describes the $\mathrm{InP} / \mathrm{InGaAs}$ DHBT process technology with the layer stack description, transistor modeling, and characteristics of the passive structures. Section III is dedicated to the circuit design, Section IV describes the measurements, and Section $\mathrm{V}$ provides the conclusions to this article.

\section{TECHNOLOGY}

\section{A. Technology Layer Description}

The circuit presented in this article is based on the Ferdinand-Braun-Institut (FBH) transferred-substrate InPDHBT technology. The cross-sectional schematic of the layer stack is shown in Fig. 1.

As shown in Fig. 1, emitter and base layers of the HBT are processed before the complete structure is flipped and bonded on a carrier silicon wafer using the in-house $\mathrm{BCB}$ bonding process. Then, the InP substrate is etched down, and collector and planarization steps are carried out. To realize the passives, SiN-based metal-insulator-metal capacitors (MIMs) with a sheet capacitance of $0.3 \mathrm{fF} / \mu \mathrm{m}^{2}$ and $\mathrm{NiCr}$-based thinfilm resistors (TFRs) with a sheet resistance of $25 \Omega / \mathrm{sq}$ are fabricated. All lithography steps are performed with a Nikon i-line Stepper. The epitaxy is industry-grade DHBT epitaxial layers on semi-insulating InP substrates. The base region is a 30-nm-thick InGaAs layer optimized for low transit time through grading the p-doping from the emitter side to the collector side (high to low). The DHBT devices exhibit $f_{t} / f_{\max }$ values of $\sim 360 / 490 \mathrm{GHz}$, respectively, and static forward current gain of $\beta \sim 30$. The breakdown voltage exceeds $4 \mathrm{~V}$ and facilitates high output power circuit design. Three gold metal layers, GD, G1, and G2 with a thickness value of $3,1.5$, and $4.5 \mu \mathrm{m}$, respectively, serve as interconnect between the circuit components. Additional details can be found in [12].

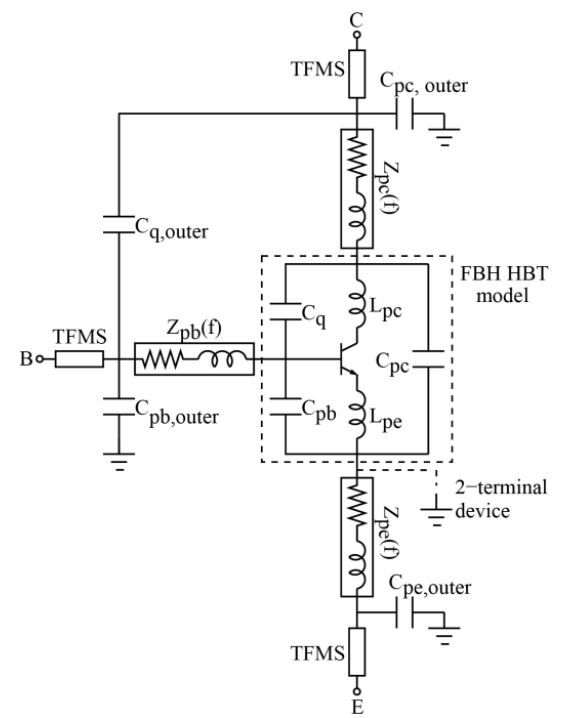

Fig. 2. Large-signal model structure for transferred substrate $\mathrm{InP} / \mathrm{InGaAs}$ DHBT. The dashed box shows the part modeled using the FBH HBT model. The emitter terminal of the FBH HBT model is directly grounded for the two-terminal device.

\section{B. Transistor Modeling}

The large-signal model structures for the transferredsubstrate $\mathrm{InP} / \mathrm{InGaAs}$ DHBTs are shown in Fig. 2. It consists of the FBH HBT model [13] embedded in an external parasitic network associated with via transitions and device electrodes. The external parasitic network is extracted using a dedicated 3-D electromagnetic (EM) simulation approach, as described in [14]. The 3-D EM simulation approach allows accurate modeling of the distribution along the device access structure and skin effect due to field penetration into the conductor. In particular, frequency-dependent impedances

$$
\begin{aligned}
Z_{p b(e, c)}(f)=\left.R_{p b(e, c)}\right|_{f \rightarrow 0}+ & \left.j 2 \pi f L_{p b(e, c)}\right|_{f \rightarrow \infty} \\
+ & R_{p b(e, c), a c} \sqrt{f}(1+j)
\end{aligned}
$$

where $f$ is the frequency, are included in series with each device terminal. Furthermore, thin-film microstrip (TFMS) lines are added to model the transition to the external interconnect environment. The parasitic network modeling approach has been verified experimentally on a small-signal model up to $220 \mathrm{GHz}$ [14]. The device in a transferred-substrate technology lends itself well to a two-terminal configuration with the emitter grounded. For added flexibility, a device in a three-terminal configuration is also available. This device requires an opening of the ground plane underneath the HBT and, thus, a modified device layout. Therefore, the large-signal model structure is modified as shown in Fig. 2 to be able to capture the effect of the modified device layout.

The FBH HBT model parameters are determined from multibias S-parameters and static $I-V$ characteristics measured over temperature. The S-parameter data have been corrected using on-wafer multiline TRL calibration setting the reference plane at the boundary of the device layout. Details in the FBH HBT model extraction procedure for transferred-substrate InP/InGaAs DHBTs can be found in [13]. 


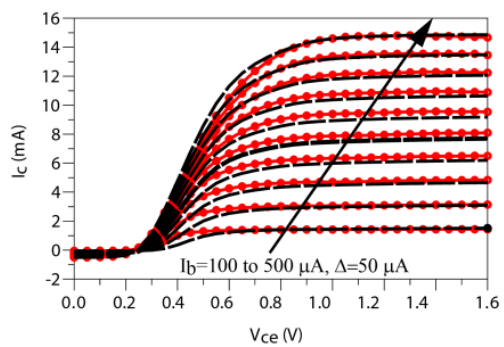

(a)

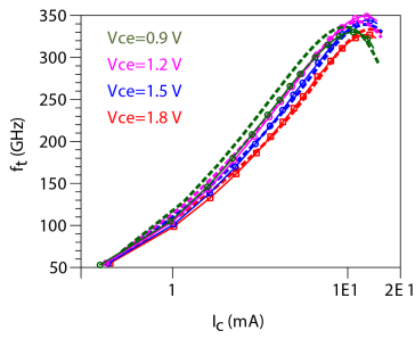

(b)

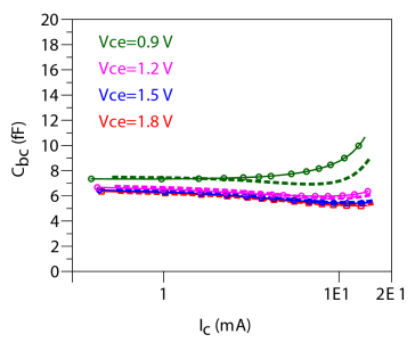

(c)
Fig. 3. Measured (dashed lines) and modeled (lines with symbols) (a) forward output characteristics at temperature $17{ }^{\circ} \mathrm{C}$, (b) unity current gain frequency versus collector current, and (c) base-collector capacitance versus collector current.

Fig. 3 shows the measured and modeled characteristics for a $0.5 \times 6 \mu \mathrm{m}^{2}$ transferred-substrate InP/InGaAs DHBT in two-terminal common-emitter configuration. The measured and modeled forward $I-V$ characteristics at $17{ }^{\circ} \mathrm{C}$ are shown in Fig. 3(a). The model fitting at other temperatures is similar. It is well known that HBTs in the $\mathrm{InP} / \mathrm{InGaAs}$ material system are prone to current blocking at the heterointerface in the collector leading to a softening of the knee region, drop in current gain, and increase in the charge storage. The FBH HBT model has no dedicated parameters to model this effect, and hence, the fitting of the softening of the knee region is only approximated, as shown in Fig. 3(a). Fig. 3(b) and (c) shows the measured and modeled unity current gain frequency, $f_{t}$, and base-collector capacitance, $C_{\mathrm{bc}}$, versus collector current with collector-emitter voltage as a parameter. In the FBH HBT model, a unified collector charge description is responsible for modeling both. Therefore, $f_{t}$ and $C_{\mathrm{bc}}$ must be fit simultaneously during the parameter extraction process. In general, the model fitting shown in Fig. 3(b) and (c) is excellent, with the exception of the lowest collector-emitter voltages where current blocking is noticeable. The delicate bias dependence of $f_{t}$ and $C_{\mathrm{bc}}$ is important for the accurate prediction of the maximum available gain/maximum stable gain (MAG/MSG) for the InP/InGaAs DHBT, which would not be possible using models dedicated to silicon-based bipolar transistors.

As a final verification of the developed transferred-substrate InP/InGaAs DHBT model, the large-signal performance measured at $94 \mathrm{GHz}$ is considered. Fig. 4(a) and (b) shows the measured and modeled large-signal performance under class- $\mathrm{A}$ operation for devices in two- and three-terminal configuration (with an externally grounded emitter), respectively. In the large-signal model for the three-terminal configuration, only the external parasitic network is changed. The model prediction of the measured large-signal performances remains

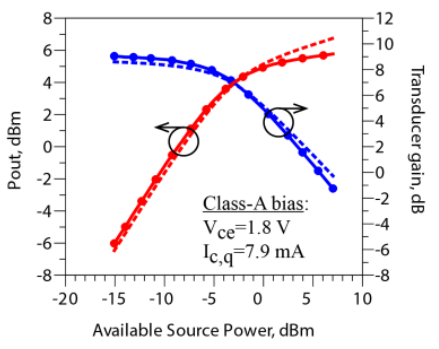

(a)

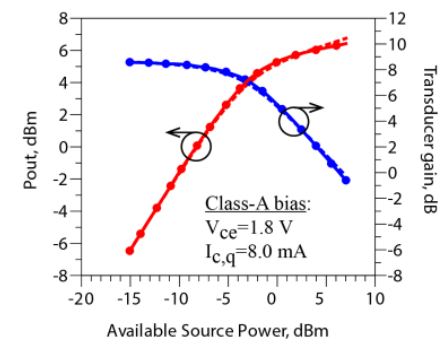

(b)
Fig. 4. Measured and modeled large-signal performance at $94 \mathrm{GHz}$ for (a) two-terminal device and (b) three-terminal device (with externally grounded emitter).
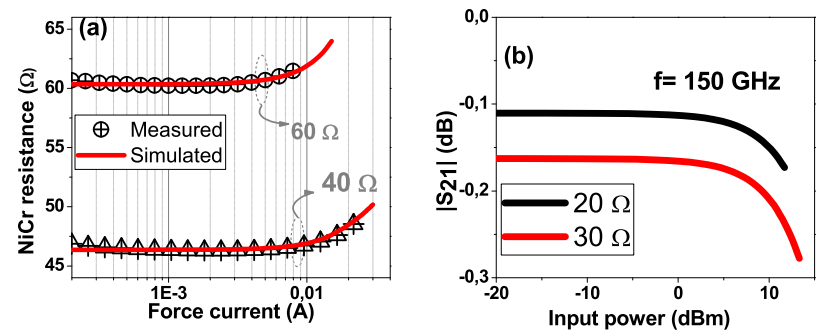

Fig. 5. (a) Measured (symbols) versus simulated (solid lines) resistance values versus force current. Increase in resistance indicates self-heating of NiCr TFRs. (b) Simulated $S_{21}$ versus input power level for a $20-\Omega$ and a $30-\Omega$ NiCr resistance.

accurate well beyond the 1-dB compression point and confirms the proposed modeling strategy.

\section{Frequency and Power Handling \\ Characteristics of the Passives}

Thermal stability due to high RF and dc currents in the passive elements of the circuit plays a pivotal role in the functionality of any $\mathrm{THz}$ MMIC. The current technology offers SiN-based MIMs and NiCr TFRs for the realization of passive networks. The MIM capacitors were designed to withstand high operating voltage way above the transistor rating, with dielectric breakdown fields reaching $1.5 \mathrm{MV} / \mathrm{cm}$.

On the other hand, the thermal stability of the $\mathrm{NiCr}$ resistors is of utmost importance to ensure long-term operation. To investigate this, a dc current injection measurement technique was employed. In this test measurement, a force current was varied and the NiCr TFR resistors were measured using a four-point contact method on a process control monitor (PCM) test structure. The output resistance was plotted versus the force current (for two nominal resistor values of 40 and $60 \Omega$ ) shown in Fig. 5(a). The results show a slight increase in the resistance value with an increase in the force current. This is an indication of the self-heating phenomena taking place in the TFR. Nevertheless, the values of the resistors show a maximum increase of $4.5 \%$ over the complete current range.

The 2-D electrothermal simulations were performed to estimate the effect on the RF performance [15]. The temperature dependence of the NiCr strip was modeled according to [16]. The resulting simulated resistance agrees with the measured values. A temperature rise of approximately $35{ }^{\circ} \mathrm{C}$ at $1 \mathrm{~mW}$ of input power was predicted by the simulations. 


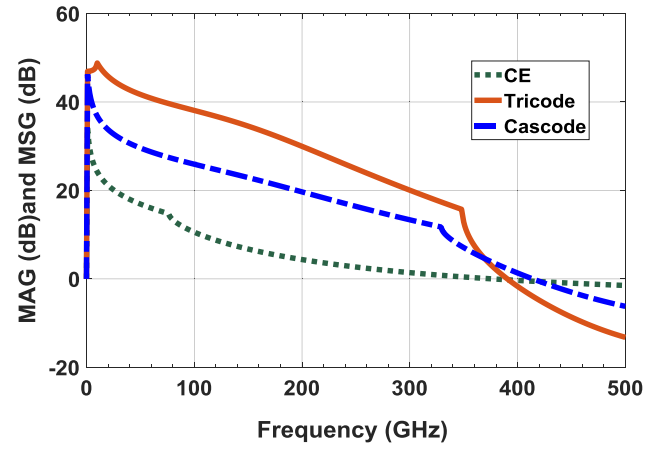

Fig. 6. Simulation of MAG and MSG for CE, cascode, and tricode subcells.

Since the upper bound of frequency is beyond $150 \mathrm{GHz}$, $\mathrm{S}$-parameters were simulated for the $\mathrm{NiCr}$ resistors versus input power to ensure the stability of the resistor from the design point of view. Considering the self-heating effects of the TFRs, small-signal parameter, $S_{21}$, was simulated using TCAD for the resistor values of 20 and $30 \Omega$ in Fig. 5(b). These two values were picked to match the similar ones used in the design later on. From Fig. 5(b), it is evident that the resistors behave ideally up to $10 \mathrm{dBm}$ of transmitted RF power. This is reasonable enough to be used on the RF path of the circuit since the maximum achievable RF output power is within this limit.

\section{Circuit AnAlysis}

\section{A. Subcell Selection for Distributed Amplifier}

Since distributed topology is a potential candidate for operating beyond $100 \%$ of the fractional $\mathrm{BW}$, the next step in the design is to choose appropriate subcell that satisfies the challenges stated in Section I. A few topologies exist as reported in the literature: common emitter (CE), cascode, stacked tricode, and buffered cascode. Each of the different subcells varies in terms of wideband property. A comparison in MAG and MSG is shown in Fig. 6.

The CE has the least gain performance among the three competitors with the tricode subcell clearly showing its advantage in terms of both MAG and MSG. Although the gain in BW is marginally higher than for the cascode, it has higher gain. This is clearly advantageous in the design of an ultrawideband DA because it directly contributes to the maximum gain-BW product of the DA.

The tricode structure can provide the best performance among the three also in terms of RF output power handling. A simplified tricode structure and its small-signal equivalent circuit are shown in Fig. 7. Since a classic cascode or tricode cell has the base directly connected to ground, an ac signal imbalance, namely, the respective $V_{\mathrm{ce}}, I_{b}$, and $I_{c}$, occurs between the $T_{C E}$ transistor and the upper transistors, $T_{\mathrm{CB} 1}$ and $T_{\mathrm{CB} 2}$. To enhance equal voltage swing across all the three transistors, a base-ground capacitor is added to each of $T_{\mathrm{CB} 1}$ and $T_{\mathrm{CB} 2}$. As a result, after a small optimization, the total voltage swing of all the three transistors can be added to provide three times the output swing. Therefore, a tricode transistor outperforms its competitors in terms of both wideband property and high RF power handling capability.

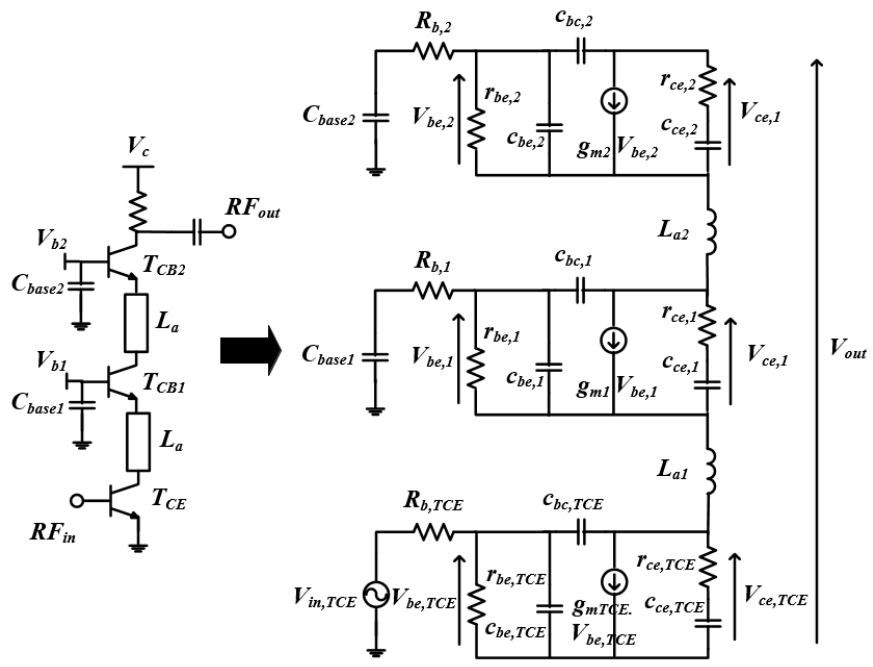

Fig. 7. Small-signal model of a tricode structure optimized with inductive peaking.

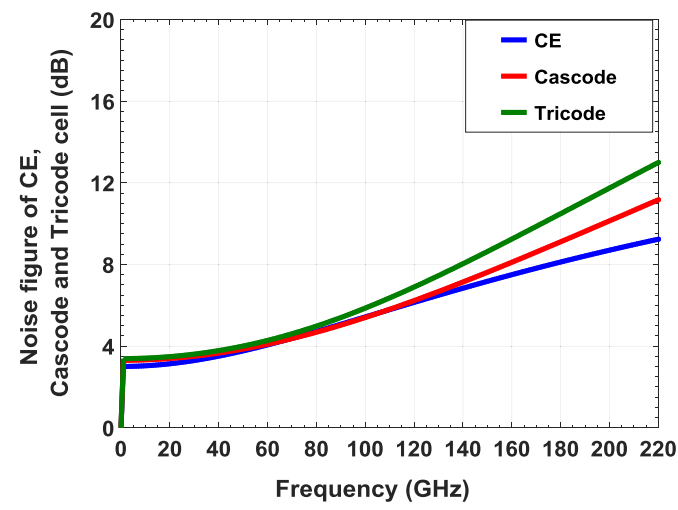

Fig. 8. Comparison of NF for a CE, cascode, and tricode subcell.

The choice of using CE, cascode, and tricode subcell can also be analyzed in terms of their respective NF versus frequency characteristics. Fig. 8 shows the NF of CE, cascode, and tricode cell, at the same dc bias conditions, i.e., the same $I_{b}, I_{c}$, and $V_{c}$ across each of the identical transistors. It is seen that NF increases when choosing tricode over a cascode or a cascode over a CE configuration. Thus, the disadvantage of using tricode is the highest NF among the three, particularly at higher frequencies of operation. The NF of cascode is higher than a CE transistor but lower than that of a tricode. Hence, it is beneficial to use a cascode over a tricode for low noise at high frequencies.

From the detailed analysis of the subcell, it is evident that the tricode offers the widest BW and highly linear performance at an expense of NF. With a choice of a 500-nm technology node, a tricode is, therefore, the optimum choice to design a DA with a BW of one-third of $f_{\max }$ of the transistor. To avoid its adverse effect on NF, the dc bias is kept low, however, just high enough to obtain a good linearity performance.

After the choice of the tricode cell, an appropriate broadband topology needs to be selected. Since the uniformly DA topology provides ultrawideband, low deviation from group delay, highly linear characteristics, and moderately low NF, this topology is applied for the amplifier design. 


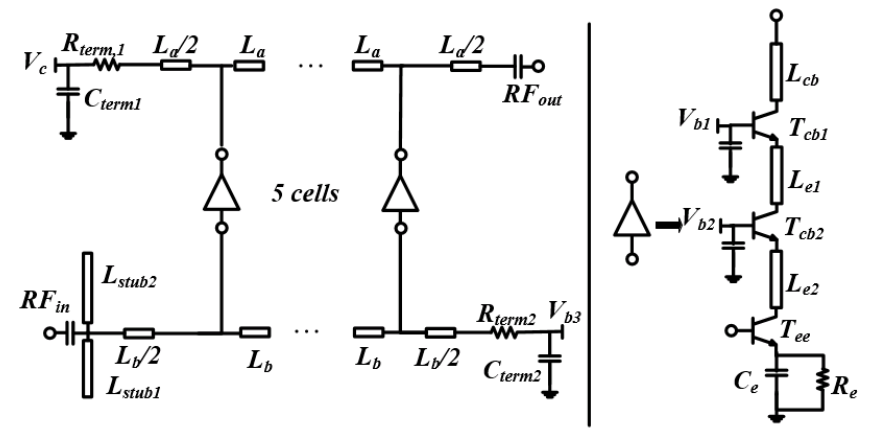

Fig. 9. Simplified schematic of the DA (detailed dc bias omitted).

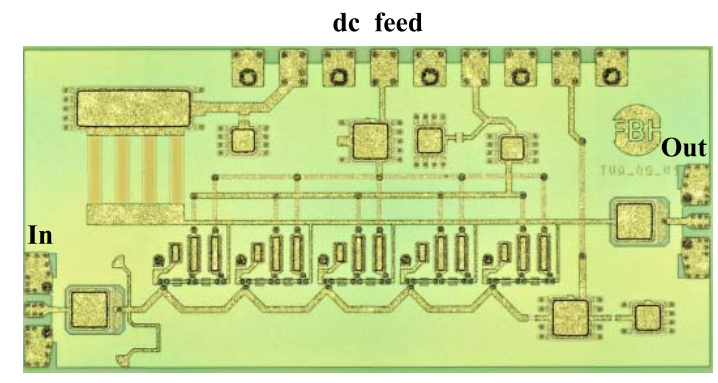

Fig. 10. Chip photograph of the tricode DA $\left(0.65 \times 1.5 \mathrm{~mm}^{2}\right)$.

\section{B. Distributed Amplifier Design With Tricode Subcell}

The simplified circuit schematic is given in Fig. 9. The circuit topology is based on a five-cell DA. Each identical cell consists of three $0.5 \times 6 \mu \mathrm{m}^{2}$ transistors arranged in the tricode configuration.

At the initial design phase, the tricode cell was optimized for maximum BW, maximum uniform gain, highest achievable linearity, and output power over the whole frequency band. To achieve this, an emitter degeneration resistance of $6.5 \Omega$ and a capacitance of $200 \mathrm{fF}$ were added to the bottom transistor $T_{\mathrm{ce}}$, as shown in Fig. 9. Between $T_{\mathrm{ce}}$ and $T_{\mathrm{cb} 1}$, and $T_{\mathrm{cb} 1}$ and $T_{\mathrm{cb} 2}$, there is an inductive transmission line, which improves the flatness of the gain characteristics at the higher end of the frequency range. Another inductive line $L_{c b}$ improves the output matching at higher frequencies.

In a second step, the entire DA is designed using the optimized cell. Since the input and output impedances of the cell are different, lines $L_{a}=210 \mu \mathrm{m}$ and $L_{b}=180 \mu \mathrm{m}$ are chosen to be of different lengths and widths, with a targeted characteristic impedance of 50 and $62 \Omega$, respectively. The termination resistances, $R_{\text {term } 1}=31.25 \Omega$ and $R_{\text {term } 2}=32 \Omega$ help to match the circuit to $50 \Omega$ at both ends. Finally, to improve the input match, two stubs are added at the input. Introduction of these stubs provides a good input match throughout the BW of operation. The fabricated circuit is shown in Fig. 10. It measures $0.65 \times 1.5 \mathrm{~mm}^{2}$.

A standalone tricode cell has high impedance looking at the output because of the reduction of Miller capacitance. From simulations, the capacitance looking at the output is $7 \mathrm{fF}$. The input impedance is much lower compared to the output with an input capacitance value of $50 \mathrm{fF}$. This capacitance values are absorbed by the input and output lines, $L_{a}$ and $L_{b}$,

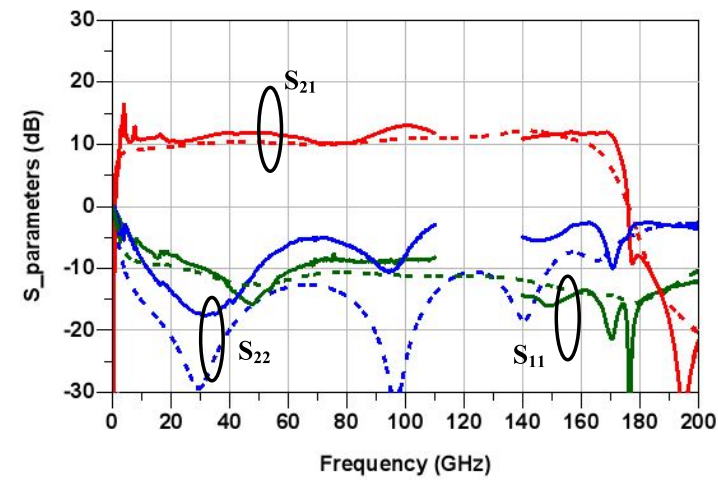

Fig. 11. Simulated (dotted line) and measured (solid lines) S-parameter of the circuit.

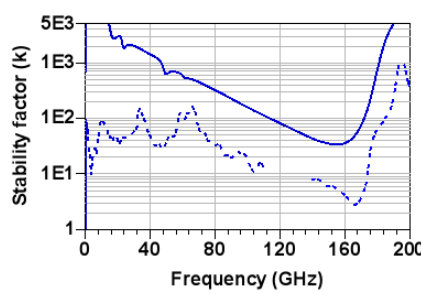

(a)

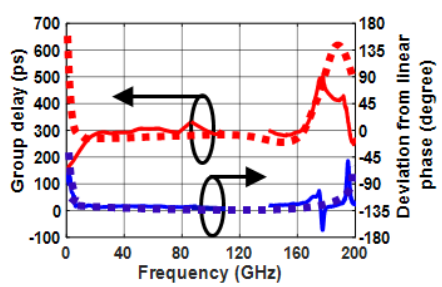

(b)
Fig. 12. Measured (dotted line) and simulated (solid line) stability factor (k) in logarithmic scale versus frequency. (b) Simulated (dotted line) and measured group delay and deviation from linear phase versus frequency.

which finally makes the input/output of DA matched to $50 \Omega$ within the BW of interest.

\section{Measurements And Discussion}

\section{A. Small-Signal Measurements}

The small-signal measurements for this circuit were performed in two steps, namely, on a $0.1-110-\mathrm{GHz}$ and a $140-220-\mathrm{GHz}$ setup. The probes for the 110- and $140-220-\mathrm{GHz}$ measurements feature pitches of 100 and $50 \mu \mathrm{m}$, respectively. An on-wafer multiline TRL calibration was applied for both setups. The carefully selected line lengths ensure the validity of the calibration over the whole frequency range. The reference impedance of the multiline TRL was transformed to $50 \Omega$ which is supported by a transmission line capacitance determination.

The results of the $\mathrm{S}$-parameter measurements are presented in Fig. 11. A forward gain $S_{21}$ of $12 \mathrm{~dB}$ was achieved with a 3 -dB BW from almost dc to $>170 \mathrm{GHz}$, with measured data available up to $110 \mathrm{GHz}$ and from 140 to $200 \mathrm{GHz}$, due to the different bands of the setup. The input of the circuit is well matched, with reflections below $-10 \mathrm{~dB}$ and a slight increase between 70 and $110 \mathrm{GHz}$. The output reflection parameter is below $-5 \mathrm{~dB}$ up to $105 \mathrm{GHz}$ and in the $140-160-\mathrm{GHz}$ frequency range. With further precise modeling of the transistors and transmission lines at these higher frequencies, $S_{22}$ could be improved further. With reference to Fig. 9, the following dc values were $V_{b 3}=1.0 \mathrm{~V}, I_{b 3}=1.2 \mathrm{~mA}, V_{b 2}=2.8 \mathrm{~V}$, $I_{b 2}=1.1 \mathrm{~mA}, V_{b 1}=3.8 \mathrm{~V}, I_{b 1}=1.4 \mathrm{~mA}$, and $V_{c}=5.9 \mathrm{~V}$, and $I_{c}=29 \mathrm{~mA}$.

As seen from the small-signal data in Fig. 12(a), the circuit is stable throughout the measured frequency region, with a 


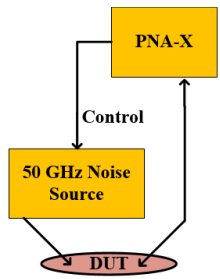

(a)

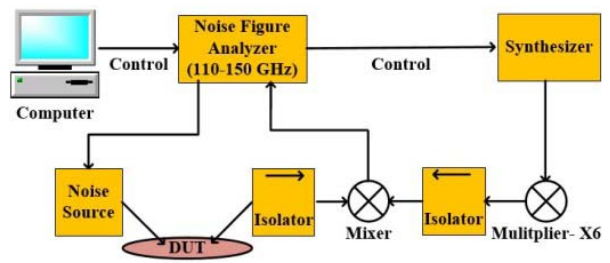

(b)
Fig. 13. (a) NF measurement setup up to $50 \mathrm{GHz}$. The noise source is needed for calibration purposes only. (b) NF measurement setup for 110-150 GHz.

worst case $k$ factor of 3 at $165 \mathrm{GHz}$, which indicates its overall stable operation. There is a low-frequency peaking in $S_{21}$ in Fig. 11, but the circuit itself is stable $(k>10)$ at these peaking frequency. Therefore, this phenomenon is attributed to the insufficient decoupling of dc feed during the S-parameter measurements. This can be eliminated using appropriate blocking capacitors at the bias feeds.

A constant group delay characteristic is highly desirable for optical communication and measurement systems. Fig. 12(b) shows the measured versus simulated group delay and the deviation from linear phase for the measured frequency ranges. The simulated group delay is uniform and matches the circuit results within the measured frequency ranges and the deviation from the linear phase is within $\pm 10^{\circ}$ throughout the measured data and increases beyond $165 \mathrm{GHz}$, which also closely matches the simulation. This confirms the circuit's excellent group delay characteristics.

\section{B. Noise Figure Measurements}

The NF measurements are added as an unexplored feature of the circuit as presented in [11]. To be used as a component in a receiver or spectroscopic application, a low NF over the whole frequency band is highly desirable. In order to investigate this further, two similar five-cell DAs were compared with respect to their NF, one using a cascode and the other with a tricode subcell, both based on the same technology node $(500-\mathrm{nm}$ InP/InGaAs DHBT). This aimed to identify how much NF degrades when using a tricode subcell instead of a cascode one.

To estimate the NF, measurements up to $50 \mathrm{GHz}$ were carried out. The setup shown in Fig. 13(a) includes a Keysight PNA-X vector network analyzer as the noise receiver and a Keysight 346CK01 50-GHz noise source for calibration purposes. Due to the built-in, dedicated noise receiver, the NF of the system was fairly low and an additional low-noise amplifier was not required.

The on-wafer NF measurements between 110 and $150 \mathrm{GHz}$ were performed at the Fraunhofer Institute IAF, Freiburg, Germany, as shown in Fig. 13(b). The setup consists of a commercial mixer module, whose local oscillator input is driven by a multiplied synthesizer signal. The NF analyzer controls the synthesizer as well as the noise source and evaluates the downconverted signals [10].

The NF measurements of the tricode-based DA for the frequency range up to 50 and $110-150 \mathrm{GHz}$ are plotted

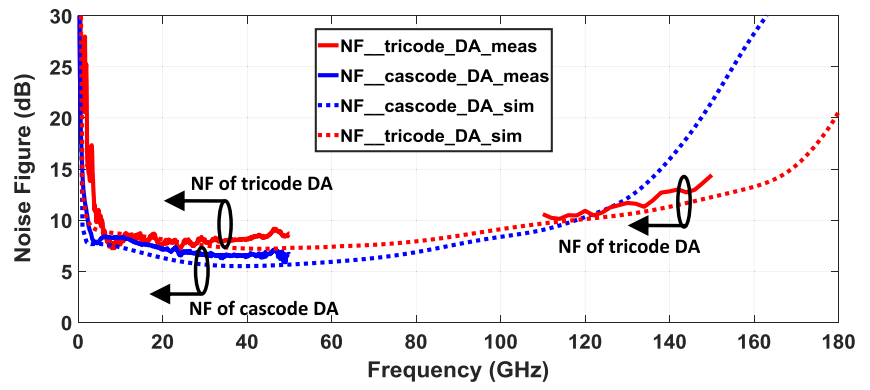

Fig. 14. Simulated (dotted) and measured (solid line) NF for the two DAs, one with a five tricode unit cells and the other with five cascode units.

in Fig. 14. The measured NF is almost constant with frequency, with an average value of $8 \mathrm{~dB}$ up to $50 \mathrm{GHz}$ and a variation of $\pm 1 \mathrm{~dB}$ within this frequency range. Between 110 and $150 \mathrm{GHz}$, the NF follows a similar trend as the simulation, going from 10 to $14.6 \mathrm{~dB}$ up to $150 \mathrm{GHz}$. This increase in $\mathrm{NF}$ is caused by its nearness to the upper limit of the BW (i.e. $175 \mathrm{GHz}$ ). Downscaling of the transistors and rightly adjusted linewidths of the amplifier will generate a higher BW and, consequently, a lower NF within this frequency range.

The NF is initially very high in the low-frequency range because of the absence of gain at very low frequencies. Lack of gain causes low SNR values which, in turn, increase the $\mathrm{NF}$ at low frequencies. The amplifier has a lower gain below $3 \mathrm{GHz}$ mainly because of low on-chip input-output decoupling capacitance $(1 \mathrm{pF})$. Higher values would increase the gain at lower frequencies, and the NF would be lower and closer to the value of $8 \mathrm{~dB}$ in the lower frequency end.

Using the same setup, NF and small-signal gain of a cascode-based DA have been measured, which is shown in Fig. 14. It is seen that the cascode-based DA offers a slightly lower NF with an average of $7.5 \mathrm{~dB}$, i.e., $0.5 \mathrm{~dB}$ better compared to its tricode counterpart. This was also illustrated by simulation in Fig. 8, which shows that the NF of a cascode subcell is slightly lower than its tricode variant.

Even though there is a slight increase of NF using the tricode unit cell in a DA, the gain-BW product is much higher. This enables certain ultrawideband optoelectronic and spectroscopic applications, where the BW offered by a simple cascode unit cell of the same technology might not be enough and the compromise in NF is acceptable.

\section{Large-Signal Measurements}

The large-signal measurements were carried out in three steps, within 5-55 GHz with a PNA-X spectrum analyzer, from 80 to $110 \mathrm{GHz}$ with an HP power meter, and within 145-165 GHz with an Erikson PM4 power meter. The measurements data were corrected by deembedding of the on-wafer probe losses in both cases.

Fig. 15 summarizes the output large-signal performance within the aforementioned frequency bands. At $150 \mathrm{GHz}$, the circuit exhibits an output power at 1-dB compression of $P_{1 \mathrm{~dB}}=8.4 \mathrm{dBm}$ and a saturated output power of $P_{\text {sat }}=10 \mathrm{dBm}$, at a maximum PAE of $6 \%$ at $150 \mathrm{GHz}$. Slightly higher values were obtained in the lower frequency 
TABLE I

StATE-OF-THE-ART DAs With LARGER THAN 110-GHz BANDWIDTH

\begin{tabular}{|c|c|c|c|c|c|c|c|c|c|}
\hline Ref. & $\begin{array}{l}\mathrm{BW}^{*} \\
(\mathrm{GHz})\end{array}$ & $\begin{array}{l}\text { Gain } \\
(\mathrm{dB})\end{array}$ & Technology & $\begin{array}{c}\text { Circuit } \\
\text { Topology }\end{array}$ & $\begin{array}{c}\text { GBP } \\
(\mathrm{GHz})\end{array}$ & $\begin{array}{c}\mathrm{P}_{\mathrm{DC}} \\
(\mathrm{mW})\end{array}$ & $\begin{array}{c}\mathrm{P}_{1 \mathrm{~dB}}(\mathrm{dBm}) \\
\text { (a) Freq }(\mathrm{GHz})\end{array}$ & $\begin{array}{c}\operatorname{Max} P A E \\
(\%)\end{array}$ & $\begin{array}{l}\text { Noise Figure } \\
\quad(\mathrm{dB})\end{array}$ \\
\hline [19] & 110 & 11 & $50 \mathrm{~nm}$ InGaAs mHEMT & Cascode & 390 & 450 & $7 @ 75$ & 1.02 & $2.5-5 @<90 \mathrm{GHz}$ \\
\hline [9] & $>110$ & 13 & $500 \mathrm{~nm}$ InP DHBT & Cascode & $>491$ & 129 & $10 @ 5-110$ & 8 & $6.5-8 @ 5-50 \mathrm{GHz}$ \\
\hline$[18]$ & $>110$ & 19.7 & $50 \mathrm{~nm}$ mHEMT & 4-stacked & $>1062$ & 541 & $10 @ 90$ & 6 & 2.5-6.4@0-98 GHz \\
\hline$[20]$ & 120 & 7.3 & $40 \mathrm{~nm}$ GaN DHFET & Cascode & 278 & 448 & $15.5 @ 20$ & 6.5 & 5-7@5-40 GHz \\
\hline [7] & 160 & 19 & $130 \mathrm{~nm} \mathrm{SiGe} \mathrm{HBT}$ & Tricode & 1515 & 560 & $9 @ 135$ & 1.4 & NA \\
\hline [5] & 170 & 10 & $130 \mathrm{~nm} \mathrm{SiGe} \mathrm{HBT}$ & Tricode & 537 & 108 & $7.5 @ 50$ & 4.9 & $6 @ 50-67 \mathrm{GHz}$ \\
\hline $\begin{array}{r}\text { This } \\
\text { work }\end{array}$ & $>170$ & 12 & 500 nmInP DHBT & Tricode & 697 & 180 & $\begin{array}{l}8.4 @ 150 / \\
6.2 @ 165 \\
\end{array}$ & $6 / 4$ & $\begin{array}{c}8 @ 5-50 \mathrm{GHz} \\
10-14.6 @ 110-150 \mathrm{GHz} \\
\end{array}$ \\
\hline$[17]$ & 180 & 18.7 & $130 \mathrm{~nm} \mathrm{SiGe} \mathrm{HBT}$ & Tricode $^{\mathrm{a}}$ & 1550 & 86 & $0 @ 100$ & 1.2 & $6 @ 60 \mathrm{GHz}$ \\
\hline [8] & $>200$ & 13.5 & $250 \mathrm{~nm}$ InP DHBT & Cascode $^{\mathrm{b}}$ & 980 & 210 & $\begin{array}{l}3.7 @ 100, \\
1.9 @ 195 \\
\end{array}$ & NA & NA \\
\hline [3] & 235 & 16 & $250 \mathrm{~nm}$ InP DHBT & Cascode $^{\mathrm{c}}$ & 1483 & 117 & NA & NA & $\begin{array}{c}9 @ 70-110 \mathrm{GHz} \\
10-12 \mathrm{~dB} @ 110-150 \mathrm{GHz}\end{array}$ \\
\hline
\end{tabular}

${ }^{*}$ Table is arranged in the order of increasing BW, ${ }^{a}$-cascaded tricode DA, ${ }^{c}$ mitter follower cascode, ${ }^{c}$-cascaded cascode DA

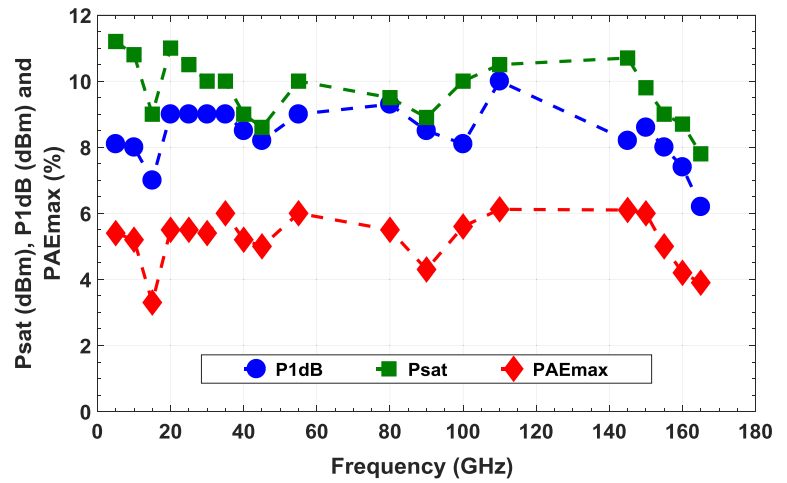

Fig. 15. Measured $P_{1 \mathrm{~dB}}, \mathrm{P}_{\mathrm{sat}}$, and $\mathrm{PAE}_{\max }$ within 5-165 GHz.

region, as expected from a DA according to the literature, with a maximum output power of $11 \mathrm{dBm}$ and $P_{1 \mathrm{~dB}}$ of $9 \mathrm{dBm}$ around $20-25 \mathrm{GHz}$, at a maximum PAE of $6 \%$. These power measurements for the lower and higher bounds of the frequency $\mathrm{BW}$ indicate that the circuit delivers uniform large-signal behavior over the whole BW. The dotted lines in Fig. 15 are only a predictive line to indicate continuous largesignal behavior. The actual measured data are plotted with symbols.

At the higher end of the frequency band, $P_{1 \mathrm{~dB}}$ is relatively constant at $8.4 \mathrm{dBm}$ up to frequencies of $150 \mathrm{GHz}$ and then drops to around $6 \mathrm{dBm}$ at $165 \mathrm{GHz}$, while the saturated output power lays $1 \mathrm{~dB}$ higher across this frequency range. Due to the decrease in linearity and saturated output power, the corresponding maximum PAE reduces from $6 \%$ to $4 \%$ when measured from 145 to $165 \mathrm{GHz}$. Power measurements at lower frequencies provided consistent data to that of the higher edge of the BW. $P_{1 \mathrm{~dB}}$ is approximately $9 \mathrm{~dB}$ between 20 and $35 \mathrm{GHz}$ and within $80-110 \mathrm{GHz}$. There are certain frequencies, namely, 15,45 , and $90 \mathrm{GHz}$, where there is a dip in the output power performance. This effect could

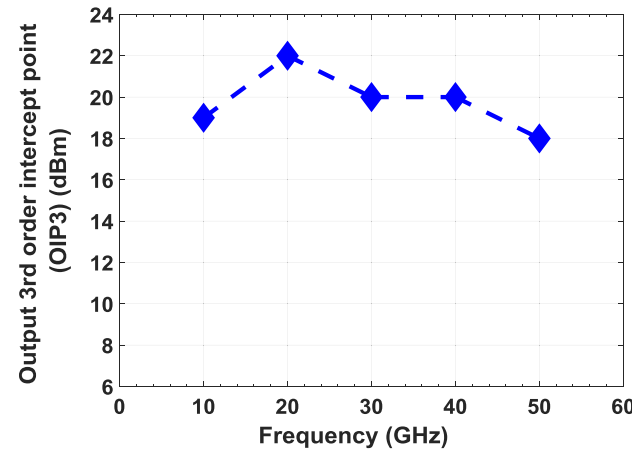

Fig. 16. Measured third-order intercept point within $10-50 \mathrm{GHz}$.

originate from the measurement system, which requires further investigation.

To further confirm the linearity of the amplifier, two-tone measurements have been performed within the frequency range of $10-50 \mathrm{GHz}$ with the help of a PNA-X. The two tones are generated directly from the PNA-X and the power of the fundamental and third-order intermodulation products are measured after calibrating out the path loss for each measurement frequency. For each fundamental tone, a second tone $1 \mathrm{MHz}$ higher in frequency was chosen and fed into the amplifier. The power of the output of the fundamental tones and third-order intermodulation products is extrapolated, which intersects at points shown in Fig. 16. Theoretically, the $1-\mathrm{dB}$ compression point, $P_{1 \mathrm{~dB}}$, is expected to lay $\sim 10 \mathrm{~dB}$ below the third-order intercept point, this graph confirms the earlier measurements on linearity in Fig. 15. The linear behavior of the circuit is, therefore, further established. Two-tone measurements in the W-band and beyond are currently limited by lack of measurement equipment.

The comparison to the state-of-the-art DAs is presented in Table I, arranged in the order of increasing BW. 
Our circuit [11] can be compared with the state-of-the-art in three aspects, namely, low deviation from linear phase within very large $\mathrm{BW}$, low NF, and highly linear characteristics even up to the higher end of the BW and correspondingly high efficiency.

Since one of the intended applications is in optical receivers, a low deviation from linear phase over the whole BW is a necessity. This article provides this feature up to $165 \mathrm{GHz}$ with a variation of $\pm 10^{\circ}$. Moreover, in receiver applications, a low NF is another necessary merit. When compared with the state of the art, the circuit provides a relatively low NF among DAs with a BW of more than $17 \mathrm{GHz}$. Despite the fact that HBT-based amplifiers have the technological disadvantage of higher NF compared to its FET-based counterparts, the circuit presented here achieves a competitive low NF within its BW. In terms of large-signal characteristics, it has the highest linearity and PAE at frequencies beyond $140 \mathrm{GHz}$ for such class of circuits. In the literature, most ultrawideband amplifiers either report a lower NF or higher linear performance, whereas our circuit performs the highest linearity with a reasonably good NF value. Only [3] and [8] show a larger BW than this article, however, at considerably lower RF output power and PAE levels. This article is the first report of an amplifier that combines all the abovementioned necessary merits in a single design. Additionally, all these merits of the circuit have been achieved at a larger technology node when compared to the others in the table. This indicates that the BW of the circuit could be further improved using a smaller InP transistor node. The circuit consumes a moderate dc power of $180 \mathrm{~mW}$, which is lower than HEMT-based devices and comparable with other HBT technologies. The circuit, therefore, can fulfill the demand when a highly linear amplifier is required with an acceptable NF value.

\section{CONCLUSION}

This article presents the characterization results of an ultrawideband DA from both small- and large-signal perspectives. The circuit achieves a $3-\mathrm{dB}$ BW of $>170 \mathrm{GHz}$. Measurements show a forward gain of $12 \mathrm{~dB}$ and a maximum deviation from the linear phase of $\pm 10^{\circ}$ up to $165 \mathrm{GHz}$. In terms of NF, the circuit exhibits a value of $8 \mathrm{~dB}$ within $5-50 \mathrm{GHz}$ and $10-14.6 \mathrm{~dB}$ within $110-150-\mathrm{GHz}$ frequency range. In terms of output power, it shows a $P_{1 \mathrm{~dB}}$ of $8.4 \mathrm{dBm}$ at $150 \mathrm{GHz}$ and a corresponding maximum PAE of $6 \%$. The measured $P_{1 \mathrm{~dB}}$ output power decreases to $P_{1 \mathrm{~dB}}=6.2 \mathrm{dBm}$ when measured at $165 \mathrm{GHz}$. At the lower frequency end, the circuit maintains its highly linear and efficient properties. Considering both small- and large-signal merits, this circuit is suitable for application in measurement systems and optical modulator and demodulators with the data rates of $200 \mathrm{~Gb} / \mathrm{s}$ and beyond.

\section{ACKNOWLEDGMENT}

The authors would like to thank the team at Fraunhofer IAF, especially $\mathrm{H}$. Massler, for their support regarding the noise measurements between 110 and $150 \mathrm{GHz}$. They would also like to thank O. Bengtsson and S. Schulz of Microwave measurements team at FBH for the two-tone measurements between 10 and $50 \mathrm{GHz}$.

\section{REFERENCES}

[1] Y. Ayasli, R. L. Mozzi, J. L. Vorhaus, L. D. Reynolds, and R. A. Pucel, "A monolithic GaAs 1-13-GHz traveling-wave amplifier," IEEE Trans. Microw. Theory Techn., vol. MTT-30, no. 7, pp. 976-981, Jul. 1982.

[2] B. Agarwal, A. E. Schmitz, J. J. Brown, M. Le, M. Lui, and M. J. W. Rodwell, "A 1-157 GHz InP HEMT traveling-wave amplifier," in IEEE Radio Freq. Integr. Circuits. (RFIC) Symp. Dig. Papers, Baltimore, MD, USA, Jun. 1998, pp. 21-23.

[3] K. Eriksson, I. Darwazeh, and H. Zirath, "InP DHBT distributed amplifiers with up to 235-GHz bandwidth," IEEE Trans. Microw. Theory Techn., vol. 63, no. 4, pp. 1334-1341, Apr. 2015.

[4] A. Arbabian and A. M. Niknejad, "A tapered cascaded multi-stage distributed amplifier with $370 \mathrm{GHz}$ GBW in $90 \mathrm{~nm}$ CMOS," in Proc. IEEE Radio Freq. Integr. Circuits (RFIC) Symp., Atlanta, GA, USA, Jun. 2008, pp. 57-60.

[5] P. V. Testa, G. Belfiore, D. Fritsche, and C. Carta, "170 GHz SiGe-BiCMOS loss-compensated distributed amplifier," IEEE J. Solid-State Circuits, vol. 50, no. 10, pp. 2228-2238, Oct. 2015.

[6] A. Arbabian and A. M. Niknejad, "A three-stage cascaded distributed amplifier with GBW exceeding $1.5 \mathrm{THz}$, in Proc. IEEE Radio Freq. Integr. Circuits (RFIC) Symp., Montreal, QC, Canada, Jun. 2012, pp. 211-214.

[7] Y. Li, W.-L. Goh, H. Tang, H. Liu, X. Deng, and Y.-Z. Xiong, "A 10 to $170 \mathrm{GHz}$ distributed amplifier using 130-nm SiGe HBTs," in Proc. Int. Symp. Integr. Circuits (ISIC), Singapore, 2016, pp. 1-4.

[8] S. Giannakopoulos, K. Eriksson, I. Darwazeh, Z. S. He, and H. Zirath, "Ultra-broadband common collector-cascode 4-cell distributed amplifier in $250 \mathrm{~nm}$ InP HBT technology with over $200 \mathrm{GHz}$ bandwidth," in Proc. 12th Eur. Microw. Integr. Circuits Conf. (EuMIC), Nuremberg, Germany, 2017, pp. 142-145.

[9] T. Shivan et al., "A highly efficient ultrawideband traveling-wave amplifier in InP DHBT technology," IEEE Microw. Wireless Compon. Lett., vol. 28, no. 11, pp. 1029-1031, Nov. 2018.

[10] T. Shivan et al., "An ultra-broadband low-noise distributed amplifier in InP DHBT technology," in Proc. 13th Eur. Microw. Integr. Circuits Conf. (EuMIC), Madrid, Spain, Sep. 2018, pp. 241-244.

[11] T. Shivan et al., "A $175 \mathrm{GHz}$ bandwidth high linearity distributed amplifier in $500 \mathrm{~nm}$ InP DHBT technology," in IEEE MTT-S Int. Microw. Symp. Dig., Boston, MA, USA, Jun. 2019, pp. 1253-1256.

[12] N. G. Weimann et al., "SciFab-A wafer-level heterointegrated InP DHBT/SiGe BiCMOS foundry process for mm-wave applications," Phys. Status Solidi A, vol. 213, no. 4, pp. 909-916, Apr. 2016.

[13] T. K. Johansen et al., "Small- and large-signal modeling of InP HBTs in transferred-substrate technology," Int. J. Microw. Wireless Technol., vol. 6, nos. 3-4, pp. 243-251, Jun. 2014.

[14] T. K. Johansen, R. Doerner, N. Weimann, M. Hossain, V. Krozer, and W. Heinrich, "EM simulation assisted parameter extraction for transferred-substrate InP HBT modeling," Int. J. Microw. Wireless Technol., vol. 10, nos. 5-6, pp. 700-708, Jun. 2018.

[15] DeckBuild 4.4.3.R, Silvaco Inc., Santa Clara, CA, USA, 2019.

[16] A. Peled, Y. Zloof, J. Farhadyan, and A. M. Peled, "A study of the temperature dependence of $\mathrm{NiCr}$ thin film resistors," Solid-State Electron., vol. 34, no. 6, pp. 667-670, Jun. 1991.

[17] D. Fritsche, G. Tretter, C. Carta, and F. Ellinger, "A trimmable cascaded distributed amplifier with $1.6 \mathrm{THz}$ gain-bandwidth product," IEEE Trans. THz Sci. Technol., vol. 5, no. 6, pp. 1094-1096, Nov. 2015.

[18] F. Thome and O. Ambacher, "A 50-nm gate-length metamorphic HEMT distributed power amplifier MMIC based on stacked-HEMT unit cells," in IEEE MTT-S Int. Microw. Symp. Dig., Honololu, HI, USA, Jun. 2017, pp. $1695-1698$.

[19] C. Zech et al., "An ultra-broadband low-noise traveling-wave amplifier based on $50 \mathrm{~nm}$ InGaAs mHEMT technology," in Proc. 7th German Microw. Conf., Ilmenau, Germany, Mar. 2012, pp. 1-4.

[20] D. F. Brown et al., "Broadband GaN DHFET traveling wave amplifiers with up to $120 \mathrm{GHz}$ bandwidth," in Proc. IEEE Compound Semiconductor Integr. Circuit Symp. (CSICS), Austin, TX, USA, Oct. 2016, pp. $1-4$. 


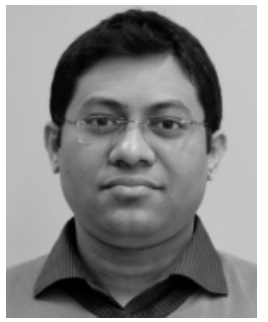

Tanjil Shivan (S'19) was born in Dhaka, Bangladesh, in 1986. He received the master's degree in electrical engineering from the Chalmers University of Technology, Gothenburg, Sweden, in 2013. He is currently pursuing the Ph.D. degree at the Ferdinand Braun Institute, Berlin, Germany.

From December 2013 to April 2014, he was a Research Assistant with the Department of Signals and Systems, Chalmers University of Technology, where he developed novel gap waveguides for 220-325-GHz frequency range. His current research interests include ultrabroadband component design in the frequency range of dc-325 GHz.

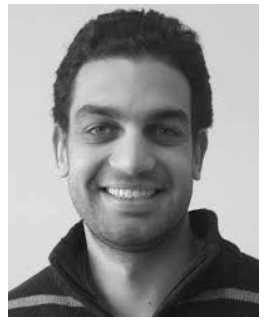

Hady Yacoub received the B.Eng. degree in electronics from the German University in Cairo, New Cairo, Egypt, in 2009, the M.Sc. degree in microelectronics from Ulm University, Ulm, Germany, in 2011, and the Ph.D. degree in electrical engineering from RWTH Aachen University, Aachen, Germany, in 2017.

In 2017, he was a Senior Researcher with the Institute of Compound Semiconductor Technology, RWTH Aachen University. He is currently the acting laboratory Director of the THz Devices Laboratory, Ferdinand-Braun Institute, Berlin, Germany, where he is involved in processing and characterization of high-frequency HBT devices.

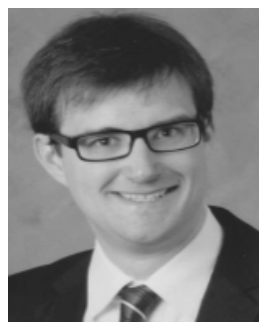

Sebastian Boppel received the Diploma degree in physics from the University of Heidelberg, Heidelberg, Germany, in 2008, and the Ph.D. degree from Goethe University, Frankfurt, Germany, in 2013.

From 2008 to 2016, he was a member of the Ultrafast Spectroscopy and Terahertz Physics Group, Goethe University. During this time, he authored or coauthored more than 50 articles, conference contributions and talks, and one book chapter on field-effect-transistor-based terahertz detection. He holds two patents. In 2016, he started working on InP-based terahertz electronics and is currently heading the InP Devices Lab at the Ferdinand-Braun-Institut, Leibniz-Institut für Höchstfrequenztechnik, Berlin, Germany

Dr. Boppel was a recipient of the Gernot-und-Carin-Frank-Prize for the best dissertation of the year

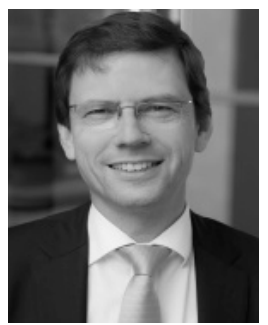

Wolfgang Heinrich (M'84-SM'95-F'09) received the Diploma, Ph.D., and Habilitation degrees from the Technical University of Darmstadt, Darmstadt, Germany, in 1983, 1987, and 1992, respectively, all in electrical engineering.

Since 1993, he has been with the FerdinandBraun-Institut (FBH), Berlin, Germany, where he is currently the Head of the Microwave Department and the Deputy Director. Since 2008, he has been a Professor with the Technical University of Berlin, Berlin. He has authored or coauthored more than 350 publications and conference contributions. His current research interests include monolithic microwave integrated circuit (MMIC) design with an emphasis on $\mathrm{GaN}$ power amplifiers, millimeter-wave integrated circuits, and electromagnetic simulation.

Dr. Heinrich has been serving the microwave community in various functions. From 2010 to 2018, he was the President of the European Microwave Association (EuMA).

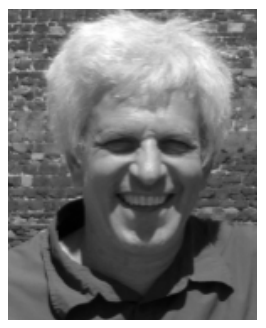

Viktor Krozer (M'91-SM'03) received the Dipl.Ing. and Dr.Ing. degrees in electrical engineering from the Technical University of Darmstadt (TU Darmstadt), Darmstadt, Germany, in 1984 and 1991, respectively.

In 1991, he became a Senior Scientist with TU Darmstadt, where he was involved in high-temperature microwave devices and circuits and submillimeter-wave electronics. From 1996 to 2002, he was a Professor with the Technical University of Chemnitz, Chemnitz, Germany. From 2002 to 2009, he was a Professor of electromagnetic systems, DTU Elektro, Technical University of Denmark, Kongens Lyngby, Denmark, where he was heading the Microwave Technology Group. From 2009 to 2012, he was an endowed Oerlikon-Leibniz-Goethe Professor of terahertz photonics with Johann Wolfgang Goethe University, Frankfurt, Germany. Since 2012, he has been heading the Goethe-Leibniz-Terahertz Center. He is also with Ferdinand-Braun-Institut (FBH), Berlin, Germany, where he is leading the Terahertz Components and Systems Group. His current research interests include terahertz electronics and imaging, monolithic microwave integrated circuit (MMIC), nonlinear circuit analysis and design, device modeling, biomedical sensors, and remote sensing instrumentation. design.

Tom K. Johansen (M'04) received the M.S. and Technical University of Denmark, Kongens Lyngby, In 1999, he joined the Electromagnetic Systems Group, DTU Elektro, Technical University of DenFrom September 2001 to March 2002, he was Visiting Scholar with the Center for Wireless Communication, University of San Diego, San Diego, CA, USA. He has spent several external research
stays with the Ferdinand-Braun-Institut (FBH), Berlin, Germany. His current

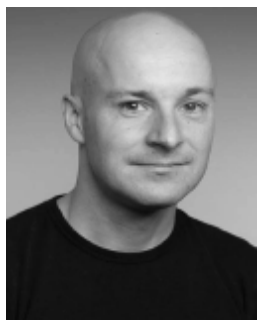

Ralf Doerner (M'97) received the Dipl.Ing. degree
in communications engineering from Technische Universität Ilmenau, Ilmenau, Germany, in 1990 . measuring techniques. In 1992, he joined the Ferdinand-Braun-Institut, Leibniz-Institut für Höchresearch interests include calibration problems in on-wafer millimeter-wave and submillimeter-wave measurements of active and passive devices and cir-
cuits, nonlinear characterization of microwave power Mr. Doerner was a recipient of the ARFTG Best Interactive Forum Paper Award. He was a co-recipient of the 2011 European Microwave Prize.

stays with the Ferdinand-Braun-Institut $(\mathrm{FBH})$, Berlin, Germany. His current
research interests include the modeling of high-frequency solid-state devices,
and microwave, millimeter-wave, and sub-millimeter-wave integrated circuit 